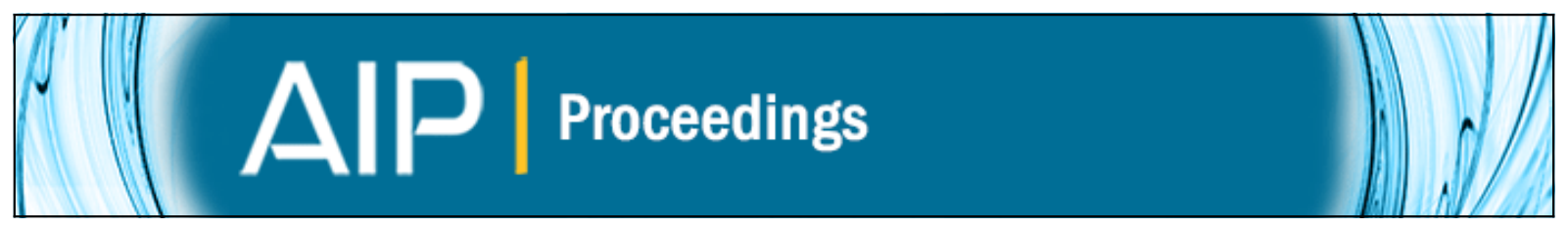

\title{
Numerical simulation and analytical solution for plane waves focused by a parabolic reflector
}

Y. T. Tsai, M. R. Haberman, and J. Zhu

Citation: AIP Conference Proceedings 1511, 421 (2013); doi: 10.1063/1.4789078

View online: http://dx.doi.org/10.1063/1.4789078

View Table of Contents: http://scitation.aip.org/content/aip/proceeding/aipcp/1511?ver=pdfcov

Published by the AIP Publishing

\section{Articles you may be interested in}

Transient axial solution for plane and axisymmetric waves focused by a paraboloidal reflector

J. Acoust. Soc. Am. 133, 2025 (2013); 10.1121/1.4794367

A numerical method for designing acoustic cloak with homogeneous metamaterials

Appl. Phys. Lett. 97, 131902 (2010); 10.1063/1.3492851

Acoustic imaging in enclosed spaces: Analysis of room geometry modifications on the impulse response J. Acoust. Soc. Am. 116, 2126 (2004); 10.1121/1.1785591

Geoacoustic inversion in range-dependent ocean environments using a plane wave reflection coefficient approach

J. Acoust. Soc. Am. 115, 1078 (2004); 10.1121/1.1646405

The low-frequency reflection and scattering of the S 0 Lamb mode from a circular through-thickness hole in a plate: Finite Element, analytical and experimental studies

J. Acoust. Soc. Am. 112, 2589 (2002); 10.1121/1.1512292 


\title{
NUMERICAL SIMULATION AND ANALYTICAL SOLUTION FOR PLANE WAVES FOCUSED BY A PARABOLIC REFLECTOR
}

\author{
Y.T. Tsai ${ }^{1}$, M. R. Haberman ${ }^{2}$, and J. Zhu ${ }^{1}$ \\ ${ }^{1}$ Department of Civil, Architectural, and Environmental Engineering, \\ The University of Texas at Austin, Austin, TX 78712 \\ ${ }^{2}$ Applied Research Laboratories \& Department of Mechanical Engineering, \\ The University of Texas at Austin, Austin, TX 78713
}

\begin{abstract}
A transient analytical solution is presented to predict the pressure responses along the axis of a parabolic reflector for normally incident plane waves. The solution was derived using geometrical acoustics and the Kirchhoff-Helmholtz integral. Results of the analytical solution were compared to the numerical simulation results, and good agreements were obtained. The numerical simulation visualizes the wave field in air to give a better understanding of propagation of the reflected waves.
\end{abstract}

Keywords: Parabolic Reflector, Plane Wave, Finite Element Analysis, Kirchhoff-Helmholtz Integral PACS: 43.20.Fn, 43.20.Px, 43.40.Dx

\section{INTRODUCTION}

Parabolic reflectors have long been used in various areas to focus plane waves. In the air-coupled impact-echo test, the airborne zero-group-velocity (ZGV) Lamb waves radiated from a resonant plate structure are quasi-planar, and the signal amplitude can effectively be enhanced by using parabolic reflectors [1-3]. Moreover, the use of parabolic reflectors further increases the signal to noise ratio (SNR) because the ambient noises are not normally incident and are not amplified equally as ZGV waves at the focal point [2]. The signal amplification at the focal point of parabolic reflectors is of great interest and has been studied intensively [4-7]. Wahlstrom [8] developed an analytical frequencydomain solution using Rayleigh integral to predict the reflected pressure field along the axis of a parabolic reflector with harmonic incident plane waves of various incident angles. The relations between focusing gain and the geometry of parabolic reflector as well as input frequency were studied. Cornet and Blackstock [9] derived a time-domain solution employing geometrical acoustics and the Kirchhoff-Helmholtz integral to predict the axial pressure response along a spherical reflector for an incident wave generated by a spark source. Hamilton [10] extended Cornet and Blackstock's solution and derived an axial transient solution for an ellipsoidal reflector with a spark source placed at one of the focal points. In this paper, a transient analytical solution is presented to calculate the pressure 


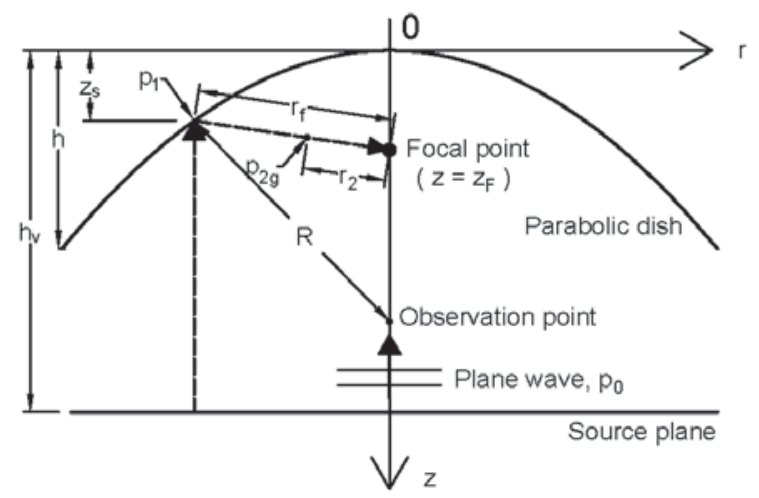

FIGURE 1. A parabolic reflector and the source plane that radiates plane waves with pressure amplitude $\mathrm{p}_{0}$. The reflector vertex is located at the origin of the coordinate $(r=z=0)$. Along the $z$ axis, the region between reflector vertex and focal point $\left(0 \leq \mathrm{z}<\mathrm{z}_{\mathrm{F}}\right)$ is considered as "before" focus, and the region from focal point to positive $z$ direction $\left(\mathrm{z}_{\mathrm{F}}<\mathrm{z}\right)$ is considered as "beyond" focus.

along the axis of a parabolic reflector for a normally incident plane wave. The derivation procedure is the same as that used by Hamilton [10] for an ellipsoidal reflector.

\section{METHODOLOGY AND RESULTS}

The analytical solution was derived using the Kirchhoff-Helmholtz integral. Geometrical acoustics was employed to determine the initial condition over the reflector surface as an input to the Kirchhoff-Helmholtz integral. Figure 1 shows the model consisting of a parabolic reflector and a source plane that radiates plane waves. The geometry of a parabolic reflector is described by $r^{2}=4 \mathrm{zZ}_{\mathrm{F}}$, where $\mathrm{Z}_{\mathrm{F}}$ is the focal length. The reflector depth is $h$, and the distance between the reflector vertex and the source plane is $h_{\mathrm{v}}$. The symbol $\mathrm{z}_{\mathrm{s}}$ denotes the $\mathrm{z}$ coordinate of a point on the reflector surface, and $r_{\mathrm{f}}$ is the distance to the focal point. $\mathrm{R}$ is the distance between the same point on surface and the observation point on the reflector axis. $\mathrm{p}_{1}$ is the pressure on the reflector surface, and $\mathrm{p}_{2 \mathrm{~g}}$ is the pressure obtained using geometrical acoustics located $r_{2}$ away from the focal point. Along the $\mathrm{z}$ axis, an observation point lies within $0 \leq \mathrm{z}<\mathrm{z}_{\mathrm{F}}$ is considered as "before" focus, and the region $\mathrm{z}_{\mathrm{F}}<\mathrm{z}$ is considered as "beyond" focus.

\section{Geometrical Acoustics And Kirchhoff-Helmholtz Integral}

To evaluate the Kirchhoff-Helmholtz integral, the pressure distribution and its inner normal derivative over the reflector surface must be known in advance. Geometrical acoustics that employs the ray theory is used to determine the initial condition over the reflector surface. Ray theory idealizes high-frequency sound waves as a group of rays and is appropriate for sound waves of $k a \gg>1$ where $k$ is the lowest wavenumber and $a$ is the minimum radius of curvature of the parabolic reflector [11]. As shown in Fig. 1, the dashed line represented a sound ray that is emitted from the source plane and reflected to the focal point after reaching the reflector surface. Assuming the pressure amplitude of an incident ray does not change before reaching the reflector surface, and there is no reflection loss on the surface, then the reflected pressure $p_{1}$ on the reflector surface is equal to $p_{0}$. Since all rays reflected from a specific $z_{\mathrm{s}}$ reach the focal point simultaneously, the reflected pressure amplitude $p_{2 \mathrm{~g}}$ along these rays can be obtained by considering the focal point as the center of a converging spherical wave. As a result, the relation between $p_{1}$ and $p_{2 \mathrm{~g}}$ for each ray may be found with $p_{1} r_{\mathrm{f}}=p_{2 \mathrm{~g}} r_{2}$, and the $p_{2 \mathrm{~g}}$ can be represented as 


$$
\frac{p_{2 g}}{p_{0}}=\frac{r_{f}}{r_{2}} f\left(t-\frac{h_{v}+z_{F}-r_{2}}{c_{0}}\right)
$$

where $f(t)$ is the function of the input wave, and $h_{v}+z_{F}-r_{2}$ is the time required for a ray to travel from the source place to a location $r_{2}$ away from the focal point. The temporal information and reflected pressure amplitude of a reflected ray can be obtained using Eq. 1. However, diffraction is not considered in geometrical acoustics and will be taken into consideration in the Kirchhoff-Helmholtz integral.

The Kirchhoff-Helmholtz integral that accounts for diffraction is used to find the reflected pressure field along the reflector axis. The Kirchhoff-Helmholtz integral can be employed to predict the pressure response at any point enclosed by a closed surface, if the pressure distribution and its inner normal derivative over this surface are known [12]. The reflected pressure $\mathrm{p}_{2}$ is calculated using the Kirchhoff-Helmholtz integral as

$$
p_{2}=\frac{1}{4 \pi} \iint_{S}\left\{\left[p_{2 g}\right] \frac{\partial}{\partial n}\left(\frac{1}{R}\right)-\frac{1}{c_{0} R} \frac{\partial R}{\partial n}\left[\frac{\partial p_{2 g}}{\partial t}\right]-\frac{1}{R}\left[\frac{\partial p_{2 g}}{\partial n}\right]\right\} d s,
$$

where $\partial / \partial n$ is the derivative in the inner direction normal to the surface, and any time argument presented in the bracket [ -$]$ is delayed by $\mathrm{R} / \mathrm{c}_{0}$. Note that the KirchhoffHelmholtz integral integrates over a closed surface, which is not the case for a parabolic reflector. However, $\mathrm{p}_{2 \mathrm{~g}}$ vanishes in the region outside of the reflector aperture, and the limit of integral is thus the edge of the reflector.

Substituting $p_{2 \mathrm{~g}}$ obtained from Eq. 1 into Eq. 2, the resulting $p_{2}$ can be written as

$$
\frac{p_{2}}{p_{0}}=H_{e}(z) f\left(\tau_{e}\right)+H_{c}(z) f\left(\tau_{c}\right)+\int_{t 1}^{t 2} H_{w}\left(z, t^{\prime}\right) f\left(t-t^{\prime}\right) d t^{\prime},
$$

where $H$ is the scaling factor for different reflected waves, $\tau$ is retarded time, and $z$ is the location of the observation point in $z$ direction. The variables in Eq. 4 are defined as

$$
\begin{aligned}
& H_{e}(z)=\frac{z_{F}\left(z+h+R_{e}\right)}{R_{e} K_{e}}, \\
& H_{c}(z)=-\frac{z_{F}\left(z+R_{c}\right)}{R_{c} K_{c}}, \\
& H_{w}\left(z, t^{\prime}\right)=-\frac{z_{F} c_{0}\left(z+z_{r}+R_{r}\right) \operatorname{sgn}\left(K_{r}\right)}{\left(z_{r}+z_{F}\right) K_{r}^{2}}, \\
& K=R+z-z_{s}-2 z_{F}, \\
& R=\sqrt{4 z_{F} z_{s}+\left(z-z_{s}\right)^{2}}, \\
& \tau=t-\frac{h_{v}-z_{s}+R}{c_{0}}, \\
& z_{r}=\frac{\left(t^{\prime} c_{0}-h_{v}\right) 2-z_{2}}{4 z_{F}-2\left(t^{\prime} c_{0}-h_{v}+z\right)}, \\
& t_{1}=\frac{h_{v}+z}{c_{0}}, \quad t_{2}=\frac{h_{v}-h+R_{e}}{c_{0}}, \quad z<z_{F},
\end{aligned}
$$




$$
t_{1}=\frac{h_{v}-h+R_{e}}{c_{0}}, \quad t_{2}=\frac{h_{v}+z}{c_{0}}, \quad z>z_{F},
$$

where the subscript $e$ indicates that the variable is associated with the reflector edge $\left(\mathrm{z}_{\mathrm{s}}=\mathrm{h}\right)$ and the subscript $c$ indicates that the variable is associated with the reflector center $\left(z_{\mathrm{S}}=0\right)$ The subscript $r$ indicates that the variable is evaluated at $\mathrm{z}_{\mathrm{s}}=\mathrm{Z}_{\mathrm{r}}$, and $\mathrm{z}_{\mathrm{r}}$ is the $\operatorname{root}$ of $\tau\left(\mathrm{z}_{\mathrm{s}}\right)=0$.

Equation 3 shows that the reflected pressure $p_{2}$ along the reflector axis consists of three reflected waves: center wave, edge wave, and wake $[10,13]$. The center wave associates with the diffraction from the reflector vertex, and the edge wave is the diffraction from the reflector edge. The wake is the reflection from the entire reflector surface due to amplitude shading along reflector surface. Equation 3 also shows that the reflected waves have the same waveform, $\mathrm{f}(\mathrm{t})$, as the incident wave. The reflected wave $\mathrm{p}_{2}$ is the linear superposition of these three waves that have different arrival times. Singularity occurs in Eq. 3 when $z=z_{F}$, and a special solution is required for predicting the focused pressure at the focal point. The solution is derived as

$$
\frac{p_{2}}{p_{0}}=\frac{2 z_{F} \ln \left(h / z_{F}+1\right)}{c_{0}} \frac{d}{d t} f\left(t-\frac{h+z_{F}}{c_{0}}\right)
$$

where $h$ is the depth of the reflector. Equation 12 indicates that the waveform of $p_{2}$ is the time derivative of the incident wave.

\section{Predicted On-Axis Results}

Three normalized pressure responses obtained at three different locations along the reflector axis are presented in Fig. 2. The input wave is numerically included in the results. The parabolic reflector has a depth of $5 \mathrm{~cm}$ and a radius of $10 \mathrm{~cm}$. The focus is located at $z=5 \mathrm{~cm}$. The distance between the reflector vertex and the source plane is $30 \mathrm{~cm}$. A onecycle sinusoidal plane wave with a period of $50 \mu \mathrm{s}(20 \mathrm{kHz})$ is used as input.

Figure 2(a) shows the normalized pressure response obtained at $3 \mathrm{~cm}$ before the focal point $(\mathrm{z}=2 \mathrm{~cm})$. In this case, the observation point is not located at the focal point, and thus the reflected waves do not arrive at the same time. The center wave travels the shortest distance to reach the observation point, while the edge wave has the longest travel distance. As a result, the center wave arrives first, and the edge wave is the last to arrive. Wake is the superposition of all the waves that are reflected from the entire reflector
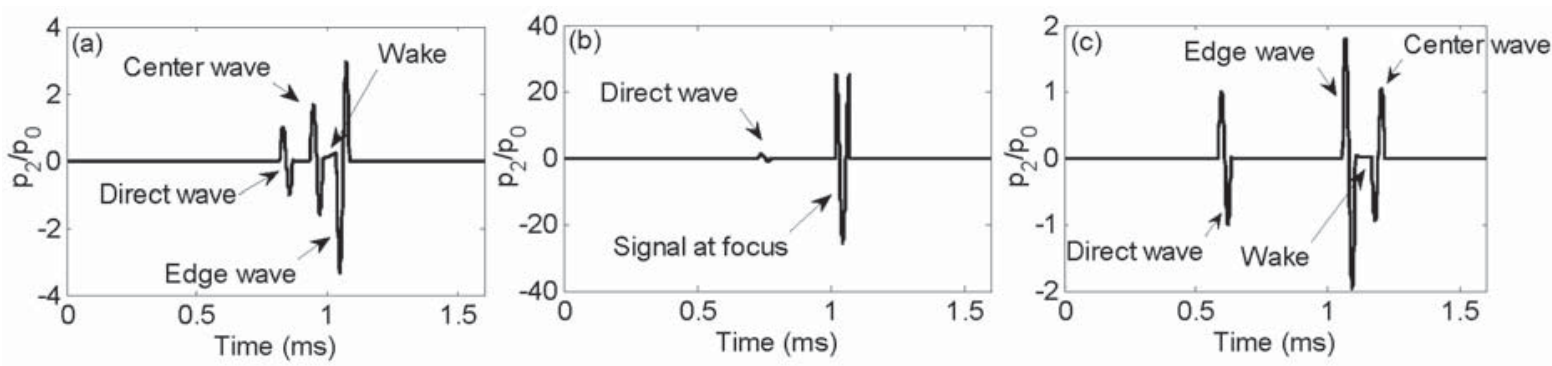

FIGURE 2. Three on-axis pressure responses are obtained at: (a) $3 \mathrm{~cm}$ before the focal point $(\mathrm{z}=2 \mathrm{~cm})$, (b) at focal point $(\mathrm{z}=5 \mathrm{~cm})$, and (c) $5 \mathrm{~cm}$ beyond the focal point $(\mathrm{z}=10 \mathrm{~cm})$. Three reflected waves (center wave, edge wave, and wake) that have the same waveform as the incident wave (direct wave) are shown. Their pressure amplitudes, phases, and arrival times vary with the location of the observation point. 
surface (from the reflector vertex to edge) and arrive in sequence. Therefore, it spans between the center wave and the edge wave as shown in Fig. 2(a). Note that the phase of the center wave is the same as that of the direct wave, and the edge wave has an opposite phase. Figure 2(b) shows the normalized pressure response obtained at the focal point. The three reflected waves arrive at the same time, and the greatest constructive interference occurs. The waveform of the resulting wave is the time derivative of the incident wave as expected. Figure 2(c) shows the normalized pressure response obtained at $5 \mathrm{~cm}$ beyond the focal point $(\mathrm{z}=15 \mathrm{~cm})$. In this case, the edge wave has the shortest distance, and the center wave has the longest among all the reflected waves. As a result, the edge wave arrives first, and the center wave is the last to arrive. The phases of the center and edge wave inverse compare to that shown in Fig. 2(a). The reason for this phase inversion is that the reflected waves converge at the focal point like a converging spherical wave and change sign after passing through the focal point.

\section{NUMERICAL VERIFICATION}

\section{$\underline{\text { FEM Model And Simulated Wave Field }}$}

A parabolic reflector with a normal incident plane wave was simulated using the finite element method (FEM) with a 2D axisymmetric model in ABAQUS. Figure 3(a) shows the model used in ABAQUS consisting of an aluminum parabolic reflector and an air section. The size of the air section is $30 \mathrm{~cm}$ by $30 \mathrm{~cm}$. The parabolic reflector vertex is located $20 \mathrm{~cm}$ away from the source plane, which is specified at the bottom of the air section. The parabolic reflector has a radius of $8 \mathrm{~cm}$ and a depth of $5 \mathrm{~cm}$, and the focal point is located $3.1 \mathrm{~cm}$ below the reflector vertex. The thickness of the reflector is $2 \mathrm{~mm}$. To properly simulate a propagating wave, the mesh size should be selected such that the wave with highest frequency is represented by at least 10 nodes [14-16]. Therefore, a mesh size of $0.5 \mathrm{~mm}$ is used in the parabolic reflector and in air in the region beneath the reflector. For the region in air that is not of interest, a larger mesh size $(1 \mathrm{~mm})$ is used. Material properties used for the aluminum reflector are $\rho=2700 \mathrm{~kg} / \mathrm{m}^{3}, \mathrm{E}=71 \mathrm{GPa}$, and $v=0.33$ [17]. Material properties used in air are $\rho=1.21 \mathrm{~kg} / \mathrm{m}^{3}$ and $\mathrm{c}_{0}=343 \mathrm{~m} / \mathrm{s}$.

Figure 3(b) shows the simulated wave field in air at $\mathrm{t}=550 \mu \mathrm{s}$. The diffracted edge wave is observed with its center located at the reflector edge. Since the model is 2D axisymmetric, the edge wave in 3D should have a donut-shaped wavefront. The wake caused by the uneven pressure distribution along the reflector surface is also observed. Figure 3(c) shows the moment
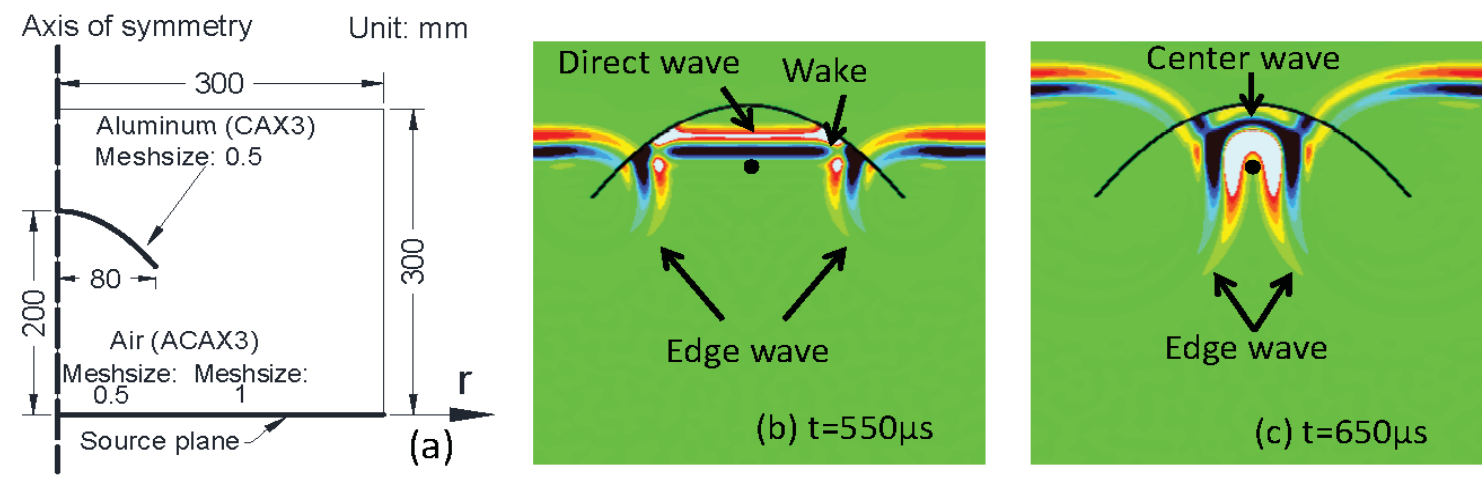

FIGURE 3. A $2 \mathrm{D}$ axisymmetric model is shown in (a). Simulated wave fields in air are obtained at (b) $\mathrm{t}=550$ $\mu \mathrm{s}$ and $(\mathrm{c}) \mathrm{t}=650 \mu \mathrm{s}$. The white arrows indicate the traveling directions of each wave. Each image consists of the original image (right half) and its mirror image (left half). The black dot represents the focal point. 

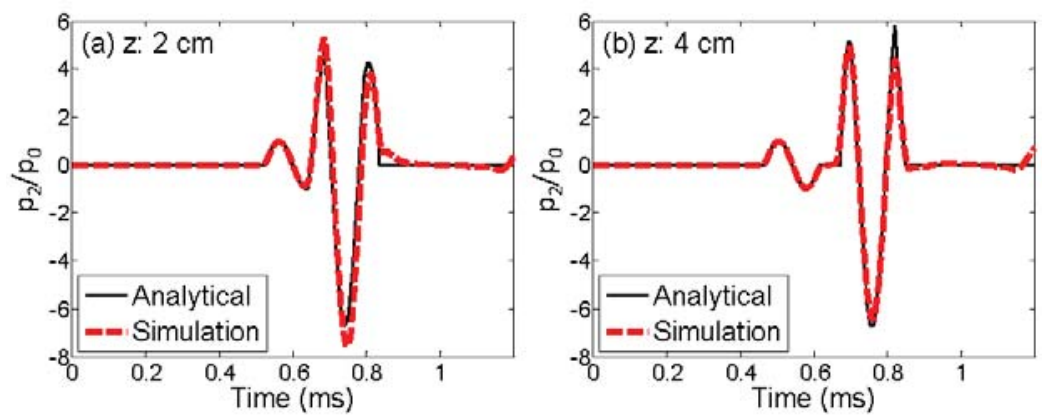

FIGURE 4. Simulation and analytical results obtained at (a) $\mathrm{z}=2 \mathrm{~cm}$, and (b) $\mathrm{z}=4 \mathrm{~cm}$. The solid lines are the analytical results, and the dashed lines are the simulation results.

before the reflected waves converge at the focal point, where a high constructive interference occurs.

\section{$\underline{\text { Axial Signal Comparison }}$}

The predicted pressure responses along the reflector axis were compared to the simulation results obtained at the same location. A longer period ( $\mathrm{T}=150 \mu \mathrm{s})$ was used for the incident plane wave described by a single cycle sinusoidal function. Given a longer wave period, the wavelength increases and the wave can be represented by more nodes. The pressure responses obtained at two locations $(\mathrm{z}=2 \mathrm{~cm}$ and $4 \mathrm{~cm})$ are presented in Fig. 4. Good agreement in both the arrival times and pressure amplitudes are obtained between the analytical and numerical results.

\section{CONCLUSION}

An analytical solution for predicting the axial pressure response along the axis of a parabolic reflector with a normal incident plane wave is presented. The solution was derived using geometrical acoustics and the Kirchhoff-Helmholtz integral. According to this solution, the reflected pressure response along the reflector axis consists of three types of waves: center wave, edge wave, and wake, and they are generated from the reflector convex, reflector edge, and the entire reflector surface, respectively. The predicted pressure response is the linear superposition of these waves having different arrival times and pressure amplitudes, which are dependent on the location of the observation point. A numerical simulation using the finite element method (FEM) was conducted to simulate the behavior of the parabolic reflector with a normal incident plane wave. A good agreement was found between the simulated and analytical signals. In addition, the wave propagation in air is visualized. The analytical solution was verified by the numerical simulations, and a better understanding of the reflected pressure response is obtained.

\section{ACKNOWLEDGEMENTS}

This work is supported by NIST Technology Innovation Program (TIP).

\section{REFERENCES}

1. S. D. Holland and D. E. Chimenti, Appl. Phys. Lett. 83, pp. 2704-2706 (2003).

2. J. Zhu and J. Popovics, J. Eng. Mech. 133, pp. 628-640 (2007). 
3. X. Dai, J. Zhu, Y.T. Tsai, and M. R. Haberman, J. Acoust. Soc. Am. 130, pp. EL167EL172 (2011).

4. H. F. Olson and I. Wolff, J. Acoust. Soc. Am. 1, pp. 410-417 (1930).

5. O. B. Hanson, J. Acoust. Soc. Am. 3, pp. 81-93 (1931).

6. R. C. Coile, J. Acoust. Soc. Am. 11, pp. 167-167 (1939).

7. R. S. Little, J. Acoust. Soc. Am. 40, pp. 919-920 (1966).

8. S. Wahlstrom, J. Audio Eng. Soc. 33, pp. 418-429 (1985).

9. E. P. Corset and D. T. Blackstock, J. Acoust. Soc. Am. 52, pp. 115-115 (1972).

10. M. F. Hamilton, J. Acoust. Soc. Am. 93, pp. 1256-1266 (1994).

11. D. T. Blackstock and C. L. Morfey, J. Acoust. Soc. Am. 89, pp. 1971-1971 (1991).

12. M. Born, E. Wolf, A. Bhatia, P. Clemmow, D. Gabor, A. Stokes, A. Taylor, P. Wayman, and W. Wilcock, Principles of Optics: Electromagnetic Theory of Propagation, Interference and Diffraction of Light, Cambridge University Press, Cambridge, 1999.

13. J. N. Tjotta and S. Tjotta, J. Acoust. Soc. Am. 71, pp. 824-834 (1982).

14. Y. C. Wang, V. Murti, and S. Valliappan, Earthq. Eng. Struct. Dyn. 21, pp. 987-1004 (1992).

15. D. Alleyne and P. Cawley, J. Acoust. Soc. Am. 89, pp. 1159-1168 (1991).

16. D. W. Zingg, H. Lomax, and H. Jurgens, SIAM J. Sci. Comput. 17, pp. 328-346 (1996).

17. D. Blackstock, Fundamentals of Physical Acoustics, Wiley-Interscience, New York, 2000. 